\title{
AVALIAÇÃO FISIOLÓGICA DE SEMENTES VISANDO PROJETOS DE RESTAURAÇÃO ECOLÓGICA COM SEMEADURA DIRETA DE ESPÉCIES NATIVAS DO CERRADO
}

\author{
Thiago Augusto Sampaio Teles ${ }^{1}$, Sybelle Barreira ${ }^{2}$
}

1 Mestre em agronegócio, Escola de Agronomia, Curso de Engenharia Florestal da Universidade Federal de Goiás (thiagosampateles@gmail.com), Goiânia, Goiás. 2 Docente da Escola de Agronomia, Curso de Engenharia Florestal da Universidade Federal de Goiás, Goiânia, Goiás.

\section{Recebido em: 06/04/2018 - Aprovado em: 10/06/2018 - Publicado em: 20/06/2018} DOI: 10.18677/EnciBio_2018A18

\begin{abstract}
RESUMO
Os desmatamentos no Cerrado não cessaram ao longo das últimas décadas, ocorrendo principalmente pela abertura de novas áreas para a agricultura e pecuária, algumas vezes abandonadas após anos de cultivo. Necessita-se restaurar estas áreas e a qualidade fisiológica de sementes é fator determinante para o sucesso da atividade. Assim, este trabalho teve por objetivo avaliar a qualidade fisiológica de sementes de Myracrodruon urundeuva Allemão., Dipteryx alata Vogel, Jacaranda cuspidifolia Mart. , Handroanthus heptaphyllus (Vell.) Mattos, Handroanthus impetiginosus (Mart. ex. DC), Hymenaea courbaril L., Qualea grandiflora Mart. e Enterolobium contortisiliquum (Vell.) Morong. (mínimo 2 matrizes), obtendo-se a taxa de germinação (\%) e umidade (\%) das sementes. Além disso, foi realizada a pesagem de mil sementes (PMS) e calculado o índice de velocidade de germinação (IVG). Para obtenção da taxa de germinação foi realizado teste com quatro repetições de 25 sementes, para cada matriz avaliada. Nas análises de umidade e PMS, seguiram-se as recomendações da RAS (2009). Os resultados dos parâmetros analisados foram submetidos a análise de variância e as diferenças entre as médias de cada matriz, avaliadas pelo teste de Tukey com nível de significância de 5\%. Os resultados obtidos para taxa de germinação e umidade variaram de $93,5 \%$ - 63,5\% e 11,2\% - 5,6\%, respectivamente e não apresentaram diferença significativa entre matrizes. $O$ mesmo pode ser observado para o IVG, cujos valores variaram de 3,96 a 1,36. Diferentemente, o PMS apresentou diferença significativa entre matrizes, sendo que a Hymenaea courbaril foi a que apresentou maior variação entre matrizes.
\end{abstract}

PALAVRAS-CHAVE: Qualidade ; Viabilidade; vigor. 


\title{
SEEDS PHYSIOLOGICAL EVALUATION AIMING ECOLOGY RESTORATION PROJECTS WITH CERRADO NATIVE SPECIES DIRECT SEEDING
}

\begin{abstract}
The deforestation in the Cerrado have not ceased over the last decades, ocurring mainly due to the starting of new agricultural and cattle raising areas, sometimes neglected after years of cultivation. It is necessary to restore those areas and the physiological quality of the seeds is a decisive fator for the success of the activity. Thus, this research aimed to evaluate the physiological quality of seeds of Myracrodruon urundeuva Allemão., Dipteryx alata Vogel, Jacaranda cuspidifolia, Handroanthus heptaphyllus (Vell.) Mattos, Handroanthus impetiginosus (Mart. ex. DC), Hymenaea courbaril L., Qualea grandiflora Mart. and Enterolobium contortisiliquum (Vell.) Morong (minimum of 2 matrices), obtaining the germination rate (\%) and humidity (\%) of the seeds. Furthermore, there have been carried out the weighing of one thousand seeds (WTS) and calculated the index of the germination speed (IVG). In order to attain the germination rate, a test with four repetitions of 25 seeds for each evaluated matrix was performed. During the humidity and PMS analyses, the RAS procedures (2009) were taken into account. The results of the analysed parameters were submitted to the analysis of variance and the diferences among the average of each matrix were evaluated by the Tukey test, with significance level of $5 \%$. The accomplished results for the germination and humidity rate varied from $93,5 \%-63,5 \%$ and $11,2 \%-5,6 \%$, respectively and did not present a significant difference among matrices. The same aspect was observed for the IVG, the values of which varied from 3,96 to 1,36. Differently, the PMS presented significant difference among matrices being the Hymenaea courbaril the one that showed the greatest variation among the matrices.
\end{abstract}

KEYWORDS: Quality; Viabilty, seed vigor.

\section{INTRODUÇÃO}

Com a necessidade de restauração de áreas ocorre uma dependência do fornecimento de sementes de qualidade, insumo básico para o sucesso dessas atividades (FIGLIOLIA et al., 2015) já que qualidade é o somatório de uma série de aspectos, tais como o fisiológico, sanitário, genético e físico. Porém, a falta de um sistema suficientemente organizado para obtenção e utilização de sementes com qualidade, limita a implementação de programas que requeiram o uso de espécies florestais nativas, em especial, aquelas sem interesse econômico imediato (FIGLIOLIA et al., 2015).

A análise de sementes é uma das formas de aferição de todo o processo produtivo e base do controle de qualidade das sementes, sendo essencial obter o diagnóstico que permite corrigir problemas nas diversas fases da produção. $\mathrm{Na}$ avaliação dos parâmetros físico, biológico e sanitário das sementes produzidas é 
possível identificar diferentes tipos de problemas e suas prováveis causas (FIGLIOLIA et al., 2015).

Alguns parâmetros da qualidade de sementes como taxa de germinação, peso de mil sementes e umidade, possuem grande variabilidade nas respostas obtidas quando se averiguam diferentes espécies e, dentro de uma mesma espécie, quanto à matriz das sementes, ao seu lote e ao tempo e diferentes métodos de armazenamento.

Isso possibilita, a comerciantes e agricultores, o fornecimento de um produto com qualidade, assim como a correta valoração das sementes de um lote e regulamentar o comércio de sementes, por contribuir para a definição e estabelecimento de padrões de qualidade. (FIGLIOLIA et al., 2015).

O local onde cada matriz se encontra pode influenciar diretamente na produção de frutos e sementes pela planta, assim fatores como: comprimento do dia, variações de temperatura, índice de pluviosidade e aspectos genéticos, exercem pressão sobre a qualidade dos frutos e sementes disponibilizados pela matriz. Avaliando matrizes de diferentes locais e ocorrendo diferença entre as matrizes, pode ser possível indicar padrões (locais de coleta) para a realização de coleta de frutos e sementes com melhor qualidade fisiológica (FIGLIOLIA et al., 2015).

Assim, o objetivo foi avaliar a qualidade fisiológica de oito espécies do Cerrado que foram selecionadas por serem utilizadas em projetos de restauração florestal, e/ou por terem importância ornamental e madeireira.

\section{MATERIAL E MÉTODOS}

As espécies utilizadas para avaliação fisiológica de sementes foram: Myracrodruon urundeuva (aroeira), Dipteryx alata (baru), Jacaranda cuspidifolia (caroba), Handroanthus heptaphyllus (ipê-roxo) Mattos, Handroanthus impetiginosus (ipê-rosa), Hymenaea courbaril (jatobá), Qualea grandiflora (pauterra) e Enterolobium contortisiliquum (tamboril). Todas as matrizes foram selecionadas para coleta de frutos e estavam localizadas no cinturão verde da Escola de Agronomia da Universidade Federal de Goiás e região próxima ao campus samambaia, em Goiânia, GO, com exceção de pau-terra cuja matriz encontra-se em Minas Gerais.

As coletas de frutos e sementes aconteceram entre os meses de julho e novembro de 2015 e foram encaminhadas ao Laboratório de Reprodução de Espécies Florestais (REFLOR) da Universidade Federal de Goiás, para beneficiamento. Todas as espécies coletadas foram imediatamente beneficiadas e logo procedeu-se as análises, não ocorrendo armazenamento das sementes. As análises de umidade, peso de mil sementes e índice de velocidade de germinação foram realizadas seguindo parâmetros da RAS. Para o teste de germinação foram realizadas quatro repetições de 25 sementes, para cada matriz avaliada. Por meio das avaliações de germinação das sementes, obteve-se o índice de velocidade de germinação (IVG), segundo Maguire (1962).

Com exceção da Aroeira que utilizou vermiculita, em todas as outras espécies foram utilizados o papel germitest como substrato. Algumas das espécies do estudo 
possuem dormência tegumentar, sendo assim, passaram por tratamento para superação de dormência, sendo utilizado o ácido sulfúrico concentrado por 30 minutos (Jatobá e tamboril).

Pau-terra e caroba necessitaram de procedimento para auxiliar o processo de germinação das sementes. A caroba foi tratada com ácido giberélico $0,3 \%$, com intuito de estimular a germinação, o uso de bioestimulantes podem interromper o período de latência, cessando ou diminuindo o impacto de fatores adversos na qualidade e desempenho das sementes.

Já o pau-terra foi tratado com nitrato de potássio $\left(\mathrm{KNO}_{3}\right)$ reagente que pode ser tanto favorável na indução como na superação da dormência para muitas espécies.

Os resultados dos testes de germinação, na quantificação da umidade, peso de mil sementes (PMS) e índice de velocidade de germinação (IVG) foram submetidos a analise de variância e as diferenças entre as médias de cada matriz foram avaliadas pelo teste de Tukey com nível de significância de $5 \%$ utilizando o programa estatístico ASSISTAT.

\section{RESULTADOS E DISCUSSÕES}

Os resultados dos parâmetros analisados estão apresentados na Tabela 1, e pode ser observado que não houve diferença significativa na taxa de germinação das sementes, o que indica alto vigor das sementes independente das matrizes. Dentre as espécies analisadas a que apresentou a maior média de taxa de germinação foi o baru seguido do Tamboril.

Dentre todos os parâmetros analisados nas oito espécies estudadas, o que apresentou maior diferença estatística significativa entre matrizes foi o peso de mil sementes, ocorrendo nas seguintes matrizes: aroeira, caroba, ipê-rosa, jatobá e pauterra. Não houve correlação entre o PMS e outros parâmetros analisados, como descritos em alguns estudos sobre qualidade fisiológica de sementes.

Para as espécies Baru, ipê-roxo e tamboril não foi realizado teste de Tukey, pois foram coletadas somente duas matrizes para essas espécies, sendo necessário para o referido teste, ao menos três valores médios.

TABELA 1. Parâmetros analisados para as oito espécies estudadas.

\begin{tabular}{cccccc}
\hline Espécie & Matriz & $\mathbf{G}(\%)$ & IVG & $\mathbf{U}(\%)$ & PMS \\
\hline \multirow{2}{*}{ Aroeira } & 01 & $77,00 \mathrm{a}$ & $3,73 \mathrm{a}$ & $11,51 \mathrm{a}$ & $17,00 \mathrm{~b}$ \\
& 02 & $76,00 \mathrm{a}$ & $3,66 \mathrm{a}$ & $10,77 \mathrm{a}$ & $16,70 \mathrm{~b}$ \\
& 03 & $91,00 \mathrm{a}$ & $4,49 \mathrm{a}$ & $11,31 \mathrm{a}$ & $17,50 \mathrm{a}$ \\
\hline & Média & 81,30 & 3,96 & 11,20 & 17,00 \\
\hline \multirow{2}{*}{ Baru } & 01 & 95,00 & 3,46 & 6,22 & $1.378,5$ \\
& 02 & 92,00 & 2,93 & 5,06 & $1.455,3$ \\
\hline \multirow{2}{*}{ Caroba } & Média & 93,50 & 3,20 & 5,64 & $1.416,9$ \\
& 01 & $84,00 \mathrm{a}$ & $3,35 \mathrm{a}$ & $7,50 \mathrm{a}$ & $30,90 \mathrm{~b}$ \\
& 02 & $73,00 \mathrm{a}$ & $2,95 \mathrm{~b}$ & $7,14 \mathrm{a}$ & $34,80 \mathrm{a}$ \\
& 03 & $90,00 \mathrm{a}$ & $3,85 \mathrm{a}$ & $7,57 \mathrm{a}$ & $34,30 \mathrm{a}$ \\
\hline & Média & 82,30 & 3,38 & 7,41 & 33,30 \\
\hline Ipê-rosa & 01 & $77,00 \mathrm{a}$ & $2,77 \mathrm{a}$ & $7,52 \mathrm{a}$ & $26,50 \mathrm{a}$ \\
& 02 & $76,00 \mathrm{a}$ & $2,31 \mathrm{a}$ & $7,51 \mathrm{a}$ & $23,00 \mathrm{c}$
\end{tabular}




\begin{tabular}{cccccc} 
& 03 & $91,00 \mathrm{a}$ & $2,38 \mathrm{a}$ & $7,74 \mathrm{a}$ & $24,00 \mathrm{~b}$ \\
\cline { 2 - 6 } & Média & 81,33 & 2,49 & 7,59 & 24,50 \\
\hline \multirow{2}{*}{ Ipê-roxo } & 01 & 67,00 & 2,02 & 7,51 & 176,70 \\
& 02 & 60,00 & 2,40 & 7,74 & 56,10 \\
\hline \multirow{3}{*}{ Jatobá } & Média & 63,50 & 2,21 & 7,63 & 116,40 \\
& 01 & $83,00 \mathrm{a}$ & $1,66 \mathrm{a}$ & $11,15 \mathrm{a}$ & $3.094,00 \mathrm{c}$ \\
& 02 & $69,00 \mathrm{a}$ & $1,20 \mathrm{~b}$ & $8,29 \mathrm{~b}$ & $5.101,70 \mathrm{a}$ \\
& 03 & $70,00 \mathrm{a}$ & $1,23 \mathrm{~b}$ & $12,04 \mathrm{a}$ & $3.921,60 \mathrm{~b}$ \\
\hline \multirow{3}{*}{ Pau-terra } & Média & 74,00 & 1,36 & 10,49 & $4.039,1$ \\
& 01 & $81,00 \mathrm{a}$ & $1,67 \mathrm{a}$ & $10,75 \mathrm{a}$ & $131,40 \mathrm{a}$ \\
& 02 & $70,00 \mathrm{a}$ & $1,47 \mathrm{a}$ & $10,87 \mathrm{a}$ & $133,80 \mathrm{a}$ \\
& 03 & $71,00 \mathrm{a}$ & $1,47 \mathrm{a}$ & $10,85 \mathrm{a}$ & $122,30 \mathrm{~b}$ \\
\hline \multirow{2}{*}{ Tamboril } & Média & 74,00 & 1,53 & 10,82 & 129,1 \\
& 01 & 83,00 & 5,03 & 4,31 & 669,20 \\
& 02 & 83,00 & 4,76 & 4,22 & 641,70 \\
& Média & 83,00 & 4,89 & 4,30 & 655,50 \\
\hline \multirow{2}{*}{ G(taxa }
\end{tabular}

G(\%) (taxa de germinação); IVG (Índice de Velocidade de Germinação); U(\%) (Umidade); PMS (Peso de Mil Sementes). As médias seguidas pela mesma letra não diferem estatisticamente entre si. Teste de Tukey ao nível de 5\% de probabilidade.

A semente de baru foi a que apresentou maior taxa de germinação com média entre as matrizes de $93,5 \%$, dados esses que corroboram com os estudos de Zuchi et al. (2016). Essa espécie possui alta taxa de germinação, devido a fatores como o fruto, que envolve a sementes protegendo de variáveis externas e também as matrizes coletadas, que se apresentaram vigorosas e localizadas em uma área em processo de restauração, que ajuda na manutenção da qualidade de frutos e sementes.

Em contraste, a espécie ipê-roxo, apresentou a menor taxa de germinação com média de $63,5 \%$, podendo ser explicado devido as péssimas condições que suas matrizes se apresentaram. Em muitas sementes foram observadas brocas causadas por algum tipo de inseto, deteriorando a semente. Os dados encontrados nas matrizes avaliadas, corroboram com os dados de Ribeiro et al. (2012) que encontraram valores médios de 63,91\% em sementes de ipê-roxo.

Outro parâmetro que pode oferecer informações sobre as características de germinação e aspectos sobre a qualidade fisiológica de sementes é o índice de velocidade de germinação. Dentre as espécies avaliadas, destaca-se o tamboril, que apresentou média de IVG de 4,89, valor esse que corrobora com a análise de Lessa et al. (2014). Fatores como o tegumento que envolve essa semente e o método de superação de dormência eficiente, favorecem a embebição e facilitam o processo de germinação, resultando em sementes com alto IVG.

Já a espécie jatobá apresentou o menor IVG $(1,36)$, além de suas matrizes terem se diferenciado estatisticamente. Apesar dessa espécie ter apresentado o menor valor de IVG entre as espécies avaliadas, os valores encontrados diferem do encontrado por Freitas et al. (2013), que apresentou valor de 0,86. Valores como os apresentados podem estar relacionados ao método de superação de dormência utilizado, pois ele interfere diretamente no processo de embebição da semente.

A umidade das sementes está intimamente associada com vários aspectos de sua qualidade fisiológica, podendo indicar o estádio de maturação, o período de colheita, influenciar no armazenamento e em eventuais tratamentos pré-germinativos. 
Além disso, conforme visto anteriormente é importante lembrar que a avaliação do estado de hidratação deve envolver não apenas a quantidade da água presente nas sementes, mas também sua atividade (BARBEDO;LAMARCA, 2015).

Quanto à umidade, as três matrizes coletadas de aroeira obtiveram a maior média $(11,19 \%)$, valores que diferem dos encontrados por Guedes et al. (2012) que observaram valor inicial de $7,96 \%$. Outra espécie que apresentou umidade alta foi pau-terra, a média entre as matrizes coletadas foi de $10,82 \%$, valores esses diferentes de Nery et al. (2014) que encontraram 32\%. Esse resultado é explicado pelo método de beneficiamento das sementes utilizados por Nery et al. (2014), que passaram por armazenamento em câmara fria, para, posteriormente, aferição da umidade.

A particularidade existente para as duas espécies citadas acima, foi que ambas possuem uma espécie de resina que foi liberada na análise de umidade, devido a alta temperatura pela qual as sementes foram expostas, alterando assim o valor de umidade devido ao aumento no peso da amostra.

A menor média de umidade encontrada foi em tamboril $(4,30 \%)$, diferindo dos resultados encontrados por Cruz et al. (2013) com valor máximo de 7,8\%. O baru também apresentou média de umidade baixa (5,64\%), diferindo dos resultados encontrados por Oliveira et al. (2014) que variou de 0 a 5,70\% utilizando diferentes métodos de análise de umidade. Aspectos estruturais como tegumento duro e forma do fruto influenciam diretamente na umidade das sementes, tanto na sua impermeabilidade, quanto na composição química, assim como o método de análise utilizado, que segue procedimentos diferentes em sementes que possuem tegumento duro.

O jatobá foi a única espécie que apresentou diferença estatística significativa entre as matrizes avaliadas para umidade, sendo que a matriz 02 foi a que apresentou o menor valor (8,29\%). Essa matriz está localizada em uma fazenda ao lado de uma estrada e isolada no meio do pasto. Enquanto que as outras duas matrizes com umidade entre 11,15 e 12,04\% estão localizadas em ambientes diferentes, uma se encontra em um fragmento florestal e outra está localizada em área com processo de restauração florestal em andamento.

O peso de mil sementes foi o parâmetro que apresentou maior variação entre as matrizes avaliadas, em cinco espécies ocorreu diferença estatística significativa, sendo o jatobá o que apresentou a maior variação. Das três matrizes avaliadas, duas apresentaram valores de 3.094 e 3.921g, enquanto que a matriz 02 apresentou peso de $5.101 \mathrm{~g}$.

Alguns estudos avaliando a qualidade fisiológica de sementes florestais (HOPPE; BRUN, 2004), indicam que o peso está diretamente ligado a umidade e outros aspectos germinativos, diferindo do que foi avaliado por esta pesquisa. Não houve correlação do PMS, com outros parâmetros analisados, ocorrendo processo inverso na espécie jatobá, a matriz que apresentou o maior valor de PMS, foi a que obteve menor umidade e taxa de germinação.

As sementes que apresentaram menor média de PMS foram aroeira e ipêrosa, 17 e 24,5g, respectivamente, apresentando também diferença estatística entre as matrizes avaliadas. É necessário aumentar a amostragem de matrizes para definir a importância do PMS como parâmetro de avaliação da qualidade, por ser um método fácil, viável e rápido, pode ser utilizado como parâmetro que ofereça homogeneidade aos lotes amostrados. 


\section{CONCLUSÃO}

- A taxa de germinação e umidade variaram de $93,5 \%-63,5 \%$ e $11,2 \%-5,6 \%$, respectivamente e não apresentaram diferença significativa entre matrizes.

- Os valores de IVG variaram de 3,96 a 1,36 , sendo o maior valor para a espécie Myracrodruon urundeuva e o menor para Hymenaea courbaril.

- O PMS apresentou diferença significativa entre matrizes, sendo que a Hymenaea courbaril foi a que apresentou maior variação entre matrizes.

\section{REFERÊNCIAS}

BARBEDO, C.J.; LAMARCA, E.V. Teor de água e dessecação. In: PINÃRODRIGUES, F.C.M.; FIGLIOLIA, M.B.; DA SILVA, A. (Orgs.) Sementes Florestais Tropicais: da ecologia á produção. Londrina-PR: ABRATES, p.308-324. 2015.

BRASIL. Ministério da Agricultura, Pecuária e Abastecimento. Regras para Análise de Sementes. Brasília: Mapa/ ACS, 2009. 399p.

CRUZ, F.R.S.; ALVES, E.U.; SILVA, R.S.; ANDRADE, L. A.; ARAÚJO, L.R. Emergência e crescimento inicial de plântulas de Enterolobium contortisiliquum (Vell.) Morong em diferentes substratos. Scientia Plena, v.9, n.12, p.01-09. 2013. Disponível em: <https://www.scientiaplena.org.br/sp/article/view/1505/925>.

FIGLIOLIA, M.B. Análise de sementes: A pesquisa e o estabelecimento de técnicas para análise de sementes florestais no Brasil. In: PINÃ-RODRIGUES, F.C.M.; FIGLIOLIA, M.B.; DA SILVA, A. (Orgs.) Sementes Florestais Tropicais: da ecologia á produção. Londrina-PR: ABRATES, p.285-307. 2015.

FREITAS, A. R.; LOPES, J. C., MATHEUS, M. T.; MENGARDA, L. H. G.; VENANCIO, L. P.; CALDEIRA, M. V. W. Superação da dormência de sementes de jatobá. Pesquisa Florestal Brasileira. Colombo, v.33, n.73, p.85-90, jan/mar. 2013. Disponível em: <https://pfb.cnpf.embrapa.br/pfb/index.php/pfb/article/view/350/294>. doi: 10.4336/2013.pfb.33.73.350

GUEDES, R. S.; ALVES, E.U.; BRUNO, R.LA.; GONÇALVES, E.P.; COSTA, E.G.; MEDEIROS, M.S. Armazenamento de sementes de Myracrodruon urundeuva Fr. All. em diferentes embalagens e ambientes. Revista Brasileira de Plantas Medicinais, v. 14, n. 01, p. 68-75, 2012. Disponível em: <http://dx.doi.org/10.1590/S1516-05722012000100010>. doi: 10.1590/S1516-05722012000100010.

HOPPE, J.M.; BRUN, E. Produção de sementes e mudas florestais. Santa Maria: UFSM, 2004. 402p. 
LESSA, B. F. T., ALMEIDA, J. P. N., PINHEIRO, C. L., NOGUEIRA, F. C. B., MEDEIROS FILHO, S. Germinação e crescimento de plântulas de Enterolobium contortisiliquum (Vell.) Morong Em função da localização da semente no fruto e regimes de temperatura. Bioscience Journal, Uberlândia, v.30, n.5, p. 1474-1483, Set./Out. 2014.

em:

<http://www.seer.ufu.br/index.php/biosciencejournal/article/view/22419>.

MAGUIRE, J.D. Speed of germination aid in selection and evaluation for seedling and vigour. Crop Science, v. 2, n. 2, p. 176-177, 1962.

NERY, M. C., DAVIDE, A. C.; SILVA, E. A. A.; SOARES, G. C. M.; NERY, F. C. Classificação fisiológica de sementes florestais quanto a tolerância à dessecação e ao armazenamento. Cerne, v.20, n.3, p.477-483. 2014. Disponível em: <http://dx.doi.org/10.1590/01047760201420031450> doi: $10.1590 / 01047760201420031450$.

OLIVEIRA, L. C.; COSTA, E.; CARDOSO, E. D.; BINOTTI, F, F. S.; JORGE, M. H. Propriedades físicas de sementes de baru em função da secagem. Revista de Agricultura Neotropical, Cassilândia-MS, v. 1, n. 1, p. 92-96, jul./set. 2014. Disponível em: <http://periodicosonline.uems.br/index.php/agrineo/article/view/223>.

RIBEIRO, C. A. D., COSTA, M. P., SENNA, D. S., CALIMAN, J.P. Fatores que afetam a germinação das sementes e a biomassa de plântulas de Tabebuia heptaphylla. Floresta, Curitiba, PR, v. 42, n.1, p.161-168, jan/mar. 2012. Disponível em: < http://dx.doi.org/10.5380/rf.v42i1.26312>. doi: 10.5380/rf.v42i1.26312

ZUCHI, J.; CAMELO, G. N.; SILVA, G. P.; GAVAZZA, M. I. A.; SALES, J. F. Testes e métodos para avaliação do potencial fisiológico de sementes de baru. Global Science and Technology, Rio Verde, v.09, n.03, p.31-38, set/dez. 2016. Disponível em: <https://rv.ifgoiano.edu.br/periodicos/index.php/gst/article/view/828/512>. 